\title{
GSTM1 Polymorphisms and Lung Cancer Risk in the Chinese Population: a Meta-Analysis Based on 47 Studies
}

\author{
Xin-Ping Chen ${ }^{1,2 \&}$, Wei-Hua $\mathrm{Xu}^{2 \&}$, Da-Feng Xü ${ }^{3}$, Xian-He Xie ${ }^{4 *}$, Jia Yao ${ }^{5}$, Sheng- \\ Miao $\mathrm{Fu}^{2 *}$
}

\begin{abstract}
Although a number of studies have been conducted on the association between GSTM1 polymorphisms and lung cancer in China, this association remains elusive and controversial. To clarify the effects of GSTM1 polymorphisms on the risk of lung cancer, a meta-analysis was performed in the Chinese population. Related studies were identified from PubMed, Springer Link, Ovid, Chinese Wanfang Data Knowledge Service Platform, Chinese National Knowledge Infrastructure (CNKI), and Chinese Biology Medicine (CBM) up to 5th April 2014. A total of 45 articles (47 studies) including 6,623 cases and 7,865 controls were involved in this meta-analysis. Overall, a significant association $(\mathrm{OR}=\mathbf{1 . 4 5 , 9 5 \%} \mathrm{CI}$ : $1.32-1.60)$ was found between the null GSTM1 and lung cancer risk when all studies in Chinese population pooled into the meta-analysis. In subgroup analyses stratified by quality score, geographic area and source of controls, the same results were observed under all the models. This meta-analysis showed that the null GSTM1 may be a potential biomarker for lung cancer risk in Chinese, but further studies with gene-gene and gene-environment interactions are required for definite conclusions.
\end{abstract}

Keywords: Meta-analysis - GSTM1 - polymorphism - lung cancer - Chinese population

Asian Pac J Cancer Prev, 15 (18), 7741-7746

\section{Introduction}

Lung cancer is the most commonly diagnosed cancer as well as the leading cause of cancer death in males globally, with 1.6 million newly confirmed cases and 1.4 million deaths from lung cancer annually (Jemal et al., 2011). Human cancers can be initiated by DNA damage caused by environmental chemical agents, such as polycyclic aromatic hydrocarbons (PAHs), and some adverse habits including tobacco smoking and alcohol use (Neumann et al., 2005).

Studies have shown that exposures to environmental and occupational PAHs are risk factors for lung cancer (Kriek et al., 1993; Li et al., 2004; Vineis \& HusgafvelPursiainen, 2005). However, not all of those who have been exposed to the risk factors will develop lung cancer, suggesting that there is individual variation in cancer susceptibility in the general population (Neumann et al., 2005). To understand the contribution of genetic variations in lung cancer, genetic association approach has been widely used and has been fruitful. For example, studies have consistently associated the development of lung cancer with the genetic factors such as glutathione S-transferase M1 (GSTM1).
The association between GSTM1 gene and lung cancer has been investigated in numerous epidemiologic studies since glutathione S-transferase was first suggested as a potential marker for susceptibility to lung cancer in 1986 (Seidegard et al., 1986). Glutathione S-transferases consist five distinct families, namely alpha (GSTA), sigma (GSTS), mu (GSTM), pi (GSTP), and theta (GSTT) (Kiyohara et al., 2002). Located on the chromosome 1p13.3, the GSTM1 plays an important role in the xenobiotics' detoxification. The most common genotype of GSTM1 gene is homozygous deletion (null genotype), which has been suggested to be associated with the loss of enzyme activity, increased vulnerability to cytogenetic damage and resulted in the increased susceptibility to cancer (Hayes et al., 2005; McIlwain et al., 2006).

Recently, the role of GSTM1 polymorphism in the etiology of different types of cancer has drawn more and more attention, including lung cancer. A number of studies in China have been conducted to explore whether GSTM1 polymorphism is associated with lung cancer susceptibility, but provided controversial or inconclusive results. Therefore, we conducted a meta-analysis to more precisely define the effect of GSTM1 polymorphism on risk for lung cancer in Chinese populations. 


\section{Materials and Methods}

\section{Search strategy}

We searched databases containing PubMed, Springer Link, Ovid, Chinese Wanfang Data Knowledge Service Platform, Chinese National Knowledge Infrastructure (CNKI), and Chinese Biology Medicine (CBM) up to 5th April 2014, using combination of the following terms: (1) GSTM1 or GST M1; (2) lung cancer or lung neoplasm or lung tumor; (3) polymorphism or variant or variation; and (4) Chinese or China. We limited the languages to English and Chinese. Besides, the references from retrieved articles were also searched.

\section{Eligibility criteria}

Studies were included in this meta-analysis if they met the following criteria: (1) case-control study or cohort study studying on associations between GSTM1 polymorphism and lung cancer susceptibility; (2) all patients with the diagnosis of lung cancer confirmed by pathological or histological examination; (3) sufficient published data about sample size, odds ratio (OR), and their $95 \%$ confidence interval (CI); (4) published in English or Chinese language; (5) all participants were Chinese. Studies were excluded when they were: (1) not case-control study or cohort study; (2) duplicate of previous publication; (3) based on incomplete data; (4) meta-analyses, letters, reviews, case reports, or editorial articles.

\section{Data extraction}

Data were independently extracted by two reviewers (Xin-ping Chen and Wei-hua Xu) using a standardized data extraction form. Discrepancies were resolved by discussion and if consensus was not achieved the decision was made by the all the reviewers. The title and abstract of all potentially relevant articles were screened to determine their relevance. Full articles were then scrutinized if the title and abstract were ambiguous. The following data were extracted from the identified studies: the first author, publication year, source of controls, geographic area, sample size, and the number of subjects with two GSTM1 genotypes. In this meta-analysis, the quality assessment of individual study was conducted according to the nine-star Newcastle-Ottawa Scale (Wells et al., 2009). For articles including different source of controls, data were extracted separately (Table 1).

\section{Statistical analysis}

Pooled odds ratios (ORs) and 95\% confidence intervals (CIs) were used to assess the association of GSTM1 genetic polymorphism with lung cancer risk in Chinese population. Given that there was distribution of null/present heterozygote in only one study selected, the Hardy-Weinberg equilibrium (HWE) test could not be conducted. Cochrane's Q test was performed to test the between-study heterogeneity. If there was heterogeneity, then the random-effects model was chosen to pool the ORs with $95 \%$ CIs, otherwise the fixed-effects model was used. Publication bias was investigated with the funnel plot, in which the Standard Error (SE) of log
OR of each study was plotted against its OR. Funnelplot asymmetry was further assessed by the method of Egger's linear regression test (Egger et al., 1997). All the $\mathrm{P}$ values were two sided. $\mathrm{P}$ value less than 0.05 was considered statistically significant. All statistical analysis was conducted by using Stata version 10.0 (Stata Corp, College Station, Texas, USA).

\section{Results}

\section{Study selection}

We identified 114 articles that examined the association between GSTM1 polymorphism and risk of Lung cancer in Chinese. However, after screening of the titles and abstracts of all 114 articles, 50 were excluded. Of the 64 potentially relevant articles identified for further assessment, one article (Wu et al., 2003) was excluded because it did not provide sufficient data about the distribution of GSTM1 genotype, 18 (Gao et al., 1998; Gao \& Zhang, 1998; Hu et al., 1998; Chen et al., 1999; Xue et al., 2001; Zhang et al., 2002; Wang et al., 2003; Gu et al., 2004; Luo et al., 2004; Ye et al., 2005; Zeng et al., 2005; Chang et al., 2006; Wang et al., 2006; Wang et al., 2007; Chang et al., 2009; Li et al., 2011; Li et al., 2012; Ma et al., 2013) were excluded because they concerned duplicate subjects. Finally, a total of 45 articles (47 studies) (Ge et al., 1996; Gao \& Zhang, 1999; Lan et al., 2000; London et al., 2000; Chen et al., 2001; Zhao et al., 2001; Chan et al., 2002; Lu et al., 2002; Zhang et al., 2002; Zhang et al., 2002a; Chen et al., 2003; Wang et al., 2003; Xian et al., 2003; Chan-Yeung et al., 2004; Li et al., 2004; Liang et al., 2004; Yang et al., 2004; Chan et al., 2005; Li et al., 2005; Li et al., 2005a; Qiao et al., 2005; Wang et al., 2005; Chen et al., 2006; Qian et al., 2006; Wang et al., 2006; Yao et al., 2006; Gu et al., 2007; Lei et al., 2007; Liu et al., 2008; Xia et al., 2008; Wang et al., 2009; Fan et al., 2010; Jin et al., 2010; Song et al., 2010; Zheng et al., 2010; Zhu, 2010; Du et al., 2011; Zhang et al., 2011; Chen et al., 2012; Han et al., 2012; Liang et al., 2012; Liu et al., 2012; Wang et al., 2012; Yao et al., 2012; Lu, 2013) containing 6,623 cases and 7,865 controls were included in this meta-analysis, and the characteristics of these studies are shown in Table 1. Among 45 included articles, 22 articles provided ethnic information, with 18 being Han, the others being Mongolian, Zhuang and Man, respectively. Unfortunately, the ethnic information of the rest 23 articles was unknown.

\section{Overall analysis}

There was evidence of between-study heterogeneity in all included studies $\left(\chi^{2}=88.54, p<0.001\right)$. Therefore, the random-effects model was used in overall analysis. The results showed that the pooled OR with $95 \%$ CI for lung cancer in Chinese with null GSTM1 was 1.45 (1.321.60) (Figure 1)

\section{Subgroup analysis}

In the subgroup analysis based on source of control, the results showed that the GSTM1 polymorphism was significantly related to lung cancer risk among populationbased population $(\mathrm{OR}=1.55,95 \% \mathrm{CI}$ : $1.39-1.73)$, as well 
Table 1. Characteristics of Studies Included in the Meta-analysis

\begin{tabular}{|c|c|c|c|c|c|c|c|c|}
\hline \multirow[t]{2}{*}{ Refence } & \multirow[t]{2}{*}{ Source of controls } & \multirow[t]{2}{*}{ Area } & \multirow[t]{2}{*}{ Case/Control } & \multicolumn{2}{|c|}{ Case } & \multicolumn{2}{|c|}{ Control } & \multirow{2}{*}{ Quality score } \\
\hline & & & & Null genotype & Non-null & Null genotype & Non-null & \\
\hline Ge 1996 & NR & Hong Kong & $89 / 25$ & 59 & 30 & 16 & 9 & 6 \\
\hline Gao 1999 & PB & Guangdong & $59 / 73$ & 34 & 25 & 36 & 37 & 7 \\
\hline Gao 1999 & HB & Guangdong & $59 / 59$ & 34 & 25 & 29 & 30 & 7 \\
\hline London 2000 & NR & Shanghai & $232 / 710$ & 122 & 110 & 427 & 283 & 8 \\
\hline Lan 2000 & PB & Yunnan & $122 / 122$ & 82 & 40 & 60 & 62 & 6 \\
\hline Chen 2001 & PB & Jiangsu & $106 / 106$ & 56 & 50 & 39 & 67 & 8 \\
\hline Zhao 2001 & HB & Singapore & $233 / 187$ & 146 & 87 & 119 & 68 & 7 \\
\hline Chan 2002 & PB & Yunnan & $56 / 99$ & 43 & 13 & 65 & 34 & 6 \\
\hline Lu 2002 & $\mathrm{~PB}$ & Beijing & $314 / 320$ & 158 & 156 & 158 & 162 & 8 \\
\hline Zhang 2002 & PB & Guangdong & $161 / 165$ & 94 & 67 & 92 & 73 & 7 \\
\hline Zhang2002a & $\mathrm{HB}$ & Jiangsu & $65 / 60$ & 41 & 24 & 27 & 33 & 7 \\
\hline Chen 2003 & $\mathrm{~PB}$ & Anhui & $38 / 99$ & 24 & 14 & 57 & 42 & 8 \\
\hline Wang 2003 & PB & Beijing+Tianjin & $164 / 181$ & 97 & 67 & 90 & 91 & 7 \\
\hline Xian 2003 & $\mathrm{HB}+\mathrm{PB}$ & Guangdong & $91 / 138$ & 56 & 35 & 73 & 65 & 8 \\
\hline Chan-Yeung 2004 & PB & Hong Kong & $229 / 197$ & 130 & 99 & 117 & 80 & 8 \\
\hline Li 2004 & $\mathrm{HB}$ & Beijing & $217 / 200$ & 127 & 90 & 95 & 105 & 8 \\
\hline Liang 2004 & $\mathrm{HB}$ & Jiangsu & $152 / 152$ & 82 & 70 & 79 & 73 & 6 \\
\hline Yang 2004 & PB & Liaoning & $186 / 139$ & 108 & 78 & 75 & 64 & 8 \\
\hline Wang 2005 & PB & Henan & $77 / 107$ & 45 & 32 & 45 & 62 & 7 \\
\hline Li 2005 & $\mathrm{HB}$ & Sichuan & $99 / 66$ & 57 & 42 & 27 & 39 & 6 \\
\hline Qiao 2005 & HB & Guangdong & $213 / 64$ & 130 & 83 & 31 & 33 & 8 \\
\hline Qiao 2005 & PB & Guangdong & $213 / 135$ & 130 & 83 & 64 & 71 & 8 \\
\hline Chan 2005 & $\mathrm{HB}$ & Hong Kong & $75 / 162$ & 31 & 44 & 91 & 71 & 6 \\
\hline Li 2005a & PB & Henan & $103 / 138$ & 63 & 40 & 61 & 77 & 7 \\
\hline Qian 2006 & PB & Tianjin & $108 / 108$ & 69 & 39 & 53 & 55 & 8 \\
\hline Wang 2006 & PB & Hubei & $56 / 42$ & 40 & 16 & 19 & 23 & 6 \\
\hline Chen 2006 & $\mathrm{~PB}$ & Hunan & $97 / 197$ & 60 & 37 & 89 & 108 & 8 \\
\hline Yao 2006 & PB & Henan & $77 / 107$ & 45 & 32 & 45 & 62 & 7 \\
\hline Lei 2007 & PB & Sichuan & $42 / 103$ & 24 & 18 & 57 & 46 & 6 \\
\hline Gu 2007 & $\mathrm{HB}+\mathrm{PB}$ & Beijing & $279 / 684$ & 164 & 115 & 325 & 359 & 8 \\
\hline Liu 2008 & $\mathrm{~PB}$ & Shandong & $110 / 125$ & 66 & 44 & 57 & 68 & 7 \\
\hline Xia 2008 & HB & Gansu & $58 / 131$ & 34 & 24 & 76 & 55 & 6 \\
\hline Wang 2009 & PB & Inner Mongolia & $304 / 310$ & 143 & 161 & 119 & 197 & 8 \\
\hline Fan 2010 & PB & Guangxi & $58 / 60$ & 40 & 18 & 33 & 27 & 6 \\
\hline Jin 2010 & HB & Anhui & $150 / 150$ & 95 & 55 & 79 & 71 & 7 \\
\hline Song 2010 & $\mathrm{~PB}$ & Shandong & $125 / 125$ & 74 & 51 & 55 & 70 & 7 \\
\hline Zhu 2010 & $\mathrm{HB}+\mathrm{PB}$ & Hunan & $160 / 160$ & 93 & 67 & 72 & 88 & 7 \\
\hline Zheng 2010 & PB & Tianjin & $265 / 307$ & 150 & 115 & 175 & 132 & 7 \\
\hline Du 2011 & HB & Sichuan & $125 / 125$ & 73 & 52 & 71 & 54 & 7 \\
\hline Zhang 2011 & PB & Yunnan & $50 / 50$ & 34 & 16 & 22 & 28 & 7 \\
\hline Han 2012 & PB & Inner Mongolia & $128 / 214$ & 79 & 49 & 89 & 125 & 6 \\
\hline Yao 2012 & $\mathrm{~PB}$ & Beijing & $150 / 150$ & 96 & 54 & 68 & 82 & 7 \\
\hline Wang 2012 & PB & Henan & $209 / 256$ & 122 & 87 & 113 & 143 & 7 \\
\hline Liu 2012 & PB & Heilongjiang & $360 / 360$ & 145 & 215 & 107 & 253 & 8 \\
\hline Chen 2012 & $\mathrm{~PB}$ & Zhejiang & $200 / 189$ & 123 & 77 & 110 & 79 & 7 \\
\hline Liang 2012 & HB & Guangxi & $68 / 70$ & 47 & 21 & 39 & 31 & 6 \\
\hline Lu 2013 & PB & Guangdong & $91 / 138$ & 61 & 30 & 70 & 68 & 7 \\
\hline
\end{tabular}

NR, not reported; HB, hospital-based; PB, population-based

Table 2. Main Results in the Total and Subgroup Analysis

\begin{tabular}{|c|c|c|c|c|c|}
\hline \multirow{2}{*}{ Subgroups } & & \multirow{2}{*}{$\begin{array}{l}\text { Random-effect model } \\
\text { OR }(95 \% \mathrm{CI})\end{array}$} & \multirow{2}{*}{$\begin{array}{l}\text { Fixed-effect model } \\
\text { OR }(95 \% \mathrm{CI})\end{array}$} & \multicolumn{2}{|c|}{ Heterogeneity } \\
\hline & & & & $\chi^{2}$ & $P$ \\
\hline Total analysis & $1.45(1.32-1.60)$ & $1.40(1.31-1.50)$ & 88.54 & $<0.001$ & \\
\hline \multirow[t]{2}{*}{ Source of control } & Population-based & $1.55(1.39-1.73)$ & $1.51(1.39-1.64)$ & 44.75 & 0.031 \\
\hline & Hospital-based & $1.27(1.04-1.57)$ & $1.26(1.08-1.46)$ & 19.38 & 0.055 \\
\hline \multirow{3}{*}{ Quality score } & 8 & $1.37(1.17-1.61)$ & $1.33(1.20-1.47)$ & 33.22 & 0.003 \\
\hline & 7 & $1.51(1.32-1.72)$ & $1.47(1.32-1.64)$ & 27.08 & 0.103 \\
\hline & 6 & $1.47(1.11-1.94)$ & $1.46(1.22-1.73)$ & 26.08 & 0.006 \\
\hline \multirow[t]{2}{*}{ Area } & South China & $1.40(1.19-1.66)$ & $1.30(1.17-1.44)$ & 54.57 & $<0.001$ \\
\hline & North China & $1.53(1.38-1.70)$ & $1.52(1.39-1.66)$ & 25.38 & 0.187 \\
\hline
\end{tabular}

as among hospital-based studies (OR $=1.26,95 \% \mathrm{CI}: 1.08$ 1.46) (Table 2). In addition, we also performed stratified analysis based on the quality score and geographic area, it revealed the similar results with all the studies (Table 2).

\section{Sensitive analysis}

To evaluate the stability of the results, we performed a sensitivity analysis by different model. All the results were not materially altered (Table 2). Hence, results of the sensitivity analysis suggest that the data in this metaanalysis are relatively stable and credible.

\section{Bias diagnosis}

The Begg's funnel plot and Egger's test were performed to access the publication bias of literatures. As showed in Figure 2, the shape of the funnel plots did 


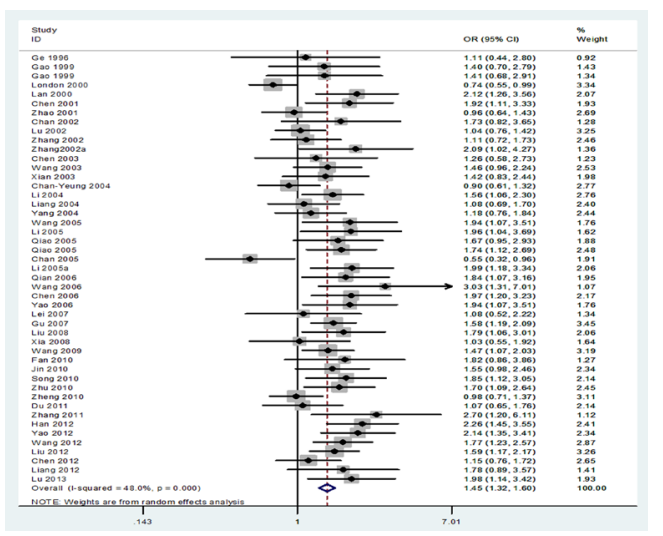

Figure 1. The Forest Plot of All Selected Studies on the Association between GSTM1 Polymorphism and Lung Cancer Risk in Chinese

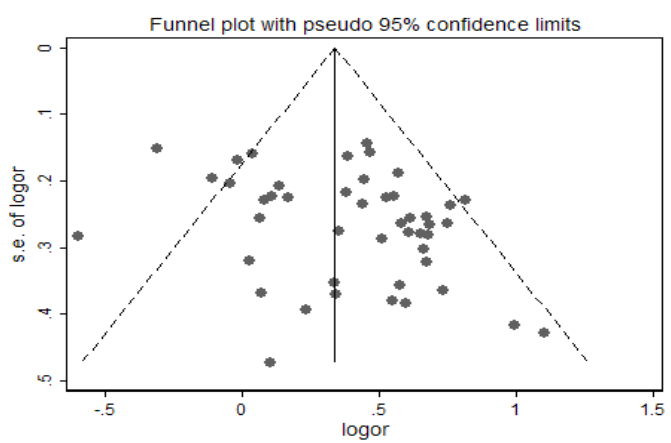

Figure 2. The Funnel plot of all Selected Studies on the Association between GSTM1 Polymorphism and Lung Cancer Risk in Chinese

reveal obvious asymmetry. Similarly, the Egger's test indicated some publication bias in the 47 reviewed studies $(\mathrm{t}=-2.51, p=0.016)$.

\section{Discussion}

Till date, a series of studies in China have focused on the relation between GSTM1 polymorphism and lung cancer risk. Nevertheless, the results were inconclusive and inconsistent. Some papers have reported that a statistically significant correlation was found between null GSTM1 and lung cancer risk. Conversely, the results from other studies suggested that the null GSTM1 was not associated with lung cancer risk. Therefore, we conducted this update meta-analysis by critically reviewing 47 individual studies on GSTM1 gene polymorphism with lung cancer risk in Chinese population. In the meta-analysis, we found that the GSTM1 null variant was significantly associated with lung cancer risk. However, our results showed a stronger association with lung cancer risk than those reported by the Carlste's study (Carlsten et al., 2008) on the GSTM1 polymorphism that included 19,638 cases and 25,266 controls of the world's overall populations $(\mathrm{OR}=1.22$, $95 \% \mathrm{CI}=1.14-1.30)$. Furthermore, our results are almost the same as those of Shi's results (Shi et al., 2008) (OR = $1.54,95 \% \mathrm{CI}=1.31-1.80)$. His report only included 2235 cases and 2315 controls of Chinese population. To our knowledge, our study represented the first meta-analysis with a large sample size on the interaction of GSTM1 variant with lung cancer in Chinese population.
When we performed stratified analyses by quality score, geographic area and source of controls, significant association with susceptibility for the development of lung cancer was found in all the subgroups. With regard to heterogeneity, some of the factors extracted in this study were the main source of heterogeneity. But it might also make attributions for other unknown factors, such as dietary habits, dinking status, other environmental exposures (passive smoking and cooking oil fume), family history of cancer, other genetic-related respiratory diseases as well as other related genetic polymorphisms.

The pathways of carcinogen metabolism are complex, mediated by the activities of multiple genes. The effect of any single gene might have a limited impact on lung cancer risk than have so far been anticipated. Many controversial data are present in literature. Positive associations were found in certain populations and not confirmed in others. In addition to an expected interethnic variability in allele frequencies, variability has also been found within an ethnic group, resulting in heterogeneity in association studies. Gene-environment interactions could be a confounding factor in these studies, with controversial findings on cancer risk. Studies taking these factors into account may eventually lead to have a better, comprehensive understanding of the association between the GSTM1 polymorphism and lung cancer risk.

This study has some limitations. First, we didn't perform subgroup analysis on smoking status and other exposure history. Second, our results were based on unadjusted estimates. Therefore, the confounding factors might influence the estimates. Third, some publication bias was detected. Because the papers included in our meta-analysis were limited to those published in either English or Chinese only in the periods between 1989 and 2014 , it is possible that some relevant published studies and unpublished studies that are likely to have null results were not included, which may have biased the results.

In summary, although studies investigating the association between GSTM1 polymorphism and the risk of lung cancer arrived at different conclusions (Piao et al., 2013; Shukla et al., 2013), this meta-analysis suggested that there was a significant association between null GSTM1 variant and lung cancer risk in the Chinese. Several recommendations on the future association studies of GSTM1-lung cancer can be made from this metaanalysis. First, a well thought-out study design is crucial for an association study. Second, it is important to make an effort to control risk factors, preferably in the design stage. Third, larger research articles in other populations with different environmental background are required. Lastly, care should be exercised in genotyping and in checking for abnormality, such as the deviation from HWP.

\section{References}

Carlsten C, Sagoo GS, Frodsham AJ, Burke W, Higgins JP (2008). Glutathione S-transferase M1 (GSTM1) polymorphisms and lung cancer: a literature-based systematic HuGE review and meta-analysis. Am J Epidemiol, 167, 759-74.

Chan EC, Lam SY, Fu KH, Kwong YL (2005). Polymorphisms of the GSTM1, GSTP1, MPO, XRCC1, and NQO1 genes 
in Chinese patients with non-small cell lung cancers: relationship with aberrant promoter methylation of the CDKN2A and RARB genes. Cancer Genet Cytogenet, 162, 10-20.

Chan Y, Wang X, Wang X, Liang Z (2002). A study of genetic polymorphism of GSTM1 gene in normal population and lung cancer population in Yunnan. J Yunnan Normal Univ, 22, 52-4.

Chan-Yeung M, Tan-Un KC, Ip MS, et al (2004). Lung cancer susceptibility and polymorphisms of glutathione-Stransferase genes in Hong Kong. Lung Cancer, 45, 155-60.

Chang F, Hu T, Wang G (2006). Relationship between CYP1A1 and GSTM1 genetic polymorphisms and lung cancer susceptibility in population of Inner Mongolia. Zhongguo Fei Ai Za Zhi, 9, 413-7.

Chang F, Wang M, Qi J, et al (2009). Genetic polymorphism of T6235C mutation in 3p non- coding region of CYP1A1 and GSTM1 genes and lung cancer susceptibility in the Mongolian population. Acad J Xi'an Jiaotong University, 21, 225-9.

Chen CM, Jin YT, Xu HY, et al (2012). Effects of CYPlAl and GSTMl gene polymorphisms and BPDE-DNA adducts on lung cancer. Zhonghua Yi Xue Yi Chuan Xue Za Zhi, 29, 23-7.

Chen HC, Cao YF, Hu WX, et al (2006). Genetic polymorphisms of phase II metabolic enzymes and lung cancer susceptibility in a population of Central South China. Dis Markers, 22, 141-52.

Chen L, Sun H, Xu M (2003). Study on the allele frequency of GSTM1 gene in normal Han population in Wannan area and the relationship between GSTM1 genotype and the risk of lung cancer. Acta Acad Med Wannan, 22, 13-6.

Chen S, Xu L, Ma J, Wu J, Xue K (1999). Identification of genetic polymorphism of CYP1A1 and GSTM1 in lung cancer patients by using allele-specific PCR and multiplex differential PCR. Carcinogenesis Teratogenesis and Mutagenesis, 11, 119-22.

Chen S, Xue K, Xu L, Ma G, Wu J (2001). Polymorphisms of the CYP1A1 and GSTM1 genes in relation to individual susceptibility to lung carcinoma in Chinese population. Mutat Res, 458, 41-7.

Du G, Ma D, Tan B, et al (2011). Relationship between genetic polymorphism ofGSTM1 gene and susceptibility to lung cancer in the population of northern Sichuan of China. Chin Clin Oncol, 16, 602-5.

Egger M, Davey Smith G, Schneider M, Minder C (1997). Bias in meta-analysis detected by a simple, graphical test. BMJ, 315, 629-34.

Fan J, Gan LG, Liang KC, Liang XM (2010). Relationship of GSTM1 and GSTT1 Genetic Polymorphisms with Lung Cancer Susceptibility in Guangxi Zhuang Population. $J$ Oncol, 16, 922-5.

Gao J \& Zhang Q (1998). Study on the relationship between GSTM1 polymorphism and lung cancer susceptibility. Carcinogenesis Teratogenesis Mutagenesis, 10, 149-51,.

Gao J, Ren C, Zhang Q (1998). CYP2D6 and GSTM1 genetic polymorphism and lung cancer susceptibility. Chin JOncol, 20, 185-6.

Gao Y,Zhang Q (1999). Study on the Polymorphisms of GSTM1 and CYP2D6 genes associated with susceptibility to lung cancer in Chinese. Chin J Public Health, 15, 488-90.

Ge H, Lam WK, Lee J, et al (1996). Analysis of L-myc and GSTM1 genotypes in Chinese non-small cell lung carcinoma patients. Lung Cancer, 15, 355-66.

Gu Y, Zhang S, Lai B, Wang H, Zhan X (2004). Relationship between genetic polymorphism of metabolizing enzymes and lung cancer susceptibility. Zhongguo Fei Ai Za Zhi, 7, 112-7.

Gu YF, Zhang ZD, Zhang SC, et al (2007). Combined effects of genetic polymorphisms in cytochrome P450s and GSTM1 on lung cancer susceptibility. Zhonghua Yi Xue Za Zhi, 87, 3064-8.

Han R, Bai Y, Chang F, et al (2012). GSTM1 gene polymorphism and lung cancer susceptibility in Man population. Central South Pharmacy, 10, 1-4.

Hayes JD, Flanagan JU, Jowsey IR (2005). Glutathione transferases. Annu Rev Pharmacol Toxicol, 45, 51-88.

$\mathrm{Hu}$ Y, Gao Y, Zhang Q (1998). Genetic polymorphisms of CYP1A1, CYP2D6 and GSTM1 related with susceptibility to lung cancer. Tumor, 18, 269-71.

Jemal A, Bray F, Center MM, Ferlay F, Ward E, Forman D (2011). Global cancer statistics. CA Cancer J Clin, 61, 69-90.

Jin Y, Xu H, Zhang C, et al (2010). Combined effects of cigarette smoking, gene polymorphisms and methylations of tumor suppressor genes on non small cell lung cancer: a hospitalbased case-control study in China. BMC Cancer, 10, 422.

Kiyohara C, Shirakawa T, Hopkin JM (2002). Genetic polymorphism of enzymes involved in xenobiotic metabolism and the risk of lung cancer. Environ Health Prev Med, 7, 47-59.

Kriek E, Van Schooten FJ, Hillebrand MJ, et al (1993). DNA adducts as a measure of lung cancer risk in humans exposed to polycyclic aromatic hydrocarbons. Environ Health Perspect, 99, 71-5.

Lan Q, He X, Costa DJ, et al (2000). Indoor coal combustion emissions, GSTM1 and GSTT1 genotypes, and lung cancer risk: a case-control study in Xuan Wei, China. Cancer Epidemiol Biomarkers Prev, 9, 605-8.

Lei FM, Li SF, Zhou WD, et al (2007). A case-control study of the impact of Glutathione S-transferase M1 polymorphism on the risk of lung cancer. Mod Prev Med, 34, 724-6.

Li D, Zhou Q, Yuan T, et al (2005). Study on the association between genetic polymorphism of CYP2E1, GSTM1 and susceptibility of lung cancer. Zhongguo Fei Ai Za Zhi, 8, 14-9.

Li W, Yue W, Zhang L, et al (2012). Polymorphisms in GSTM1, CYP1A1, CYP2E1, and CYP2D6 are associated with susceptibility and chemotherapy response in non-small-cell lung cancer patients. Lung, 190, 91-8.

Li WY, Lai BT, Zhan XP (2004). The relationship between genetic polymorphism of metabolizing enzymes and the genetic susceptibility to lung cancer. Zhonghua Liu Xing Bing Xue Za Zhi, 25, 1042-5.

Li Y, Chen J, Gao Y (2011). Influence of smoking and the polymorphisms of CYP1A1 and GSTM1 on the susceptibility of lung cancer. J Practical Diagnosis and Therapy, 25, 140-3.

Li Y, Tang X, Ma X, Chen J (2005). Glutathione S- transferaseM1 Polymorphisms and Susceptibility to Lung cancer. J Med Forum, 26, 10-2.

Liang G, Pu Y, Yin L (2004). Studies of the Genes Related to Lung Cancer Susceptibility in Nanjing Han Population, China. Hereditas (Beijing), 26, 584-8.

Liang K, Gan L, Ruan Z, et al (2012). Correlation research of the relationship between the genetic polymorphism of GSTM1 and GSTT1 in the Zhuang population and lung cancer. Acta Med Sinica, 25, 813-7.

Liu D, Wang F, Wang Q, et al (2012). Association of glutathione S-transferase M1 polymorphisms and lung cancer risk in a Chinese population. Clin Chim Acta, 414, 188-90.

Liu Q, Liu J, Song B, Wang ZH (2008). Relationship between susceptibility to lung lancer and genetic polymorphism in CYP1A1 and GSTM1. Shandong Med J, 48, 32-4.

London SJ, Yuan JM, Chung FL, et al (2000). Isothiocyanates, glutathione S-transferase M1 and T1 polymorphisms, and lung-cancer risk: a prospective study of men in Shanghai, 
China. Lancet, 356, 724-9.

Lu KQ (2013). Study on the association between GSTMl gene polymorphism and lung cancer. Capital Med, 12, 25-7.

Lu W, Xing D, Qi J, et al (2002). Genetic polymorphism in myeloperoxidase but not GSTM1 is associated with risk of lung squamous cell carcinoma in a Chinese population. Int J Cancer, 102, 275-9.

Luo C, Chen Q, Cao W, Chen S (2004). Combined analysis of polymorphisms of GSTM1 and mutations of p53 gene in the patients with lung cancer. Chin J Clin Oncol, 31, 1218-20.

Ma D, Du G, Tan B, et al (2013). Study on genetic polymorphism of GSTM1 and GSTT1 related with susceptibility to lung cancer in the Population of Northern Sichuan of China. Sichuan J Cancer Control, 26, 136-9.

McIlwain CC, Townsend DM, Tew KD (2006). Glutathione S-transferase polymorphisms: cancer incidence and therapy. Oncogene, 25, 1639-48.

Neumann AS, Sturgis EM, Wei QY (2005). Nucleotide excision repair as a marker for susceptibility to tobacco-related cancers: a review of molecular epidemiological studies. $\mathrm{Mol}$ Carcinog, 42, 65-92.

Piao JM, Shin MH, Kim HN, et al (2013). Glutathione-Stransferase (GSTM1, GSTT1) null phenotypes and risk of lung cancer in a Korean population. Asian Pac J Cancer Prev, 14, 7165-9.

Qian B, Han H, Gu F, et al (2006). Case-Control Study Genetic Polymorphism in CYP1A1 and GSTM1 and Smoking and Susceptibility to Lung Cancer. Chin J Clin Oncol, 33, 500-2.

Qiao GB, Sun CS, Li LS, Zeng WS, Jiang RC (2005). A casecontrol study on relationship between absence of GSTM1 gene, smoking and susceptibility to non-small cell lung cancer. J Fourth Mil Med Univ, 26, 1008-10.

Seidegard J, Pero RW, Miller DG, et al (1986). A glutathione transferase in human leukocytes as a marker for the susceptibility to lung cancer. Carcinogenesis, 7, 751-3.

Shi X, Zhou S, Wang Z, Zhou Z, Wang Z (2008). CYP1A1 and GSTM1 polymorphisms and lung cancer risk in Chinese populations: a meta-analysis. Lung Cancer, 59, 155-63.

Shukla RK, Tilak AR, Kumar C, et al (2013). Associations of CYP1A1, GSTM1 and GSTT1 polymorphisms with lung cancer susceptibility in a Northern Indian population. Asian Pac J Cancer Prev, 14, 3345-9.

Song B, Liu J, Huang H, et al (2010). Effects of metabolic enzyme CYP1A1 and GSTM1 gene polymorphisms and smoking factors on the occurrence of male lung squamous cell carcinoma. Basic \& Clin Med, 30, 1193-6.

Vineis P \& Husgafvel-Pursiainen K (2005). Air pollution and cancer: biomarker studies in human populations. Carcinogenesis, 26, 1846-55.

Wang D, Chen S, Wang B, Cai X, Xian X (2006). A case control study on relationship between lung cancer and genetic polymorphism of CYP1A1, CYP2E1 and GSTM1 in Han Nationality, in Guangzhou Area. Bull Chin cancer, 15, 579-82.

Wang D, Chen S, Wang B, Zhou W (2007). Interaction between smoking and polymorphism of GST in lung cancer. Chin Prev Med, 8, 466-7.

Wang J, Deng Y, Cheng J, Ding J, Tokudome S (2003). GST genetic polymorphisms and lung adenocarcinoma susceptibility in a Chinese population. Cancer Lett, 201, 185-93.

Wang J, Deng Y, Li L, et al (2003). Association of GSTM1, CYP1A1 and CYP2E1 genetic polymorphisms with susceptibility to lung adenocarcinoma: a case-control study in Chinese population. Cancer Sci, 94, 448-52.

Wang MJ, Chang FH, Yin Q, Qi J, Wang G (2009). Relationship of GSTM1 polymorphism and lung cancer susceptibility in
Mongolian population. Chin J Public Health, 25, 1447-8.

Wang N, Wu YJ, Zhou XL, Wu YM (2012). Association between genetic polymorphism of metabolizing enzymes and DNA repairing enzymes and the susceptibility of lung cancer in Henan population. J Hygiene Res, 41, 251-6.

Wang N, Zhuang D, Wu Y (2005). Research on relationship between GSTM1 gene deletion and lung cancer genetic susceptibility. J Henan Univ Sci Tech, 23, 7-8.

Wang Q, Lu Q, Zhen H, Bao M, Zhang H (2006). Relationship between CYP2C9 and GST M1 genetic polymorphism and lung cancer susceptibility. Cancer Res Prev Treat, 33, 8-10.

Wells GA, Shea B, O'Connell D, et al (2009). The NewcastleOttawa Scale (NOS) for assessing the quality if nonrandomized studies in meta-analyses. Available from: www.ohri.ca/programs/clinical_epidemiology/oxford.htm.

Wu CL, Chen SD, Lin GP, et al (2003). A case-control study on relationship between polymorphisms of CYP1A1, GSTM1 and lung cancer. South China J Prev Med, 29, 13-6.

Xia Y, Sun QF, Shang B, et al (2008). Polymorphisms of the cytochrome P450 and glutathione S-transferase genes associated with lung cancer susceptibility for the residents in high radon-exposed area. Chin J Radiol Med Prot, 28, 327-32.

Xian X, Chen S, Wang B (2003). The relationship between polymorphism of GSTM1 and susceptibility to lung cancer. Pract Prev Med, 10, 635-7.

Xue K, Xu L, Chen S, Ma J, Wu J (2001). Polymorphisms of the CYP1A1 and GSTM1 genes and their combined effects on individual susceptibility to lung cancer in a Chinese population. Zhonghua Yi Xue Yi Chuan Xue Za Zhi, 18, 125-7.

Yang XR, Wacholder S, Xu Z, et al (2004). CYP1A1 and GSTM1 polymorphisms in relation to lung cancer risk in Chinese women. Cancer Lett, 214, 197-204.

Yao W, Wang N, Wu YJ, Wu YM (2006). Relationshipbetween deletion of GSTM1, GSTT1 genes and susceptibility to lung cancer. Chin J Public Health, 22, 1070-72.

Yao Z, E Y, Wang H (2012). The interacted effects between glutathione s-transferase gene polymorphism and smoking in lung cancer. Chin J Med Guide, 14, 185-6.

Ye W, Chen S, Chen Q (2005). Interaction between serum selenium level and polymorphism of GSTM1 in lung cancer. Acta Nutrimenta Sinica, 27, 17-20.

Zeng M, Chen S, Xie C, et al (2005). Case control study on relationship between lung cancer and its susceptibility marker. Chin J Public Health, 21, 771-4.

Zhang J, Hu Y, Hu C, Wang S (2002). Relationship between genetic polymorphisms of GSTM1 as well as GSTT1 and lung cancer. Chin J Pathophysiology, 18, 352-5.

Zhang J, Hu Y, Hu C, Wang S (2002a). Study on genetic polymorphisms of GSTM1 and GSTT1 related with inherent susceptibility to lung cancer in women. Chin J Public Health, 18, 273-5.

Zhang J, Long X, Xiong G, et al (2011). The Relationship between Glutathione S-transferase M1 and Susceptibility t o Xuanwei's Lung Cancer. J Kunming Med Univ, 32, 56-8.

Zhang L, Wang X, Hao X, Shi Y, Liu Z (2002). Relationship between susceptibility to lung cancer and genetic polymorphism in P4501A1, GSTM1. Chin J Clin Oncol, 29, 536-40.

Zhao B, Seow A, Lee EJ, et al (2001). Dietary isothiocyanates, glutathione S-transferase-M1,-T1 polymorphisms and lung cancer risk among Chinese women in Singapore. Cancer Epidemiol Biomarkers Prev, 10, 1063-7.

Zheng D, Hua F, Mei C, Wan H, Zhou Q (2010). Association between GSTM1 genetic polymorphism and lung cancer risk by SYBR green I Real-time PCR Assay. Zhongguo Fei Ai Za Zhi, 13, 506-10 